\title{
Erratum to: Phase II metabolism of the soy isoflavones genistein and daidzein in humans, rats and mice: a cross-species and sex comparison
}

\author{
Sebastian T. Soukup ${ }^{1} \cdot$ Jussi Helppi $^{2}$ - Dennis R. Müller ${ }^{3}$ Oliver Zierau ${ }^{4}$ • \\ Bernhard Watzl ${ }^{5} \cdot$ Günter Vollmer $^{4} \cdot$ Patrick Diel $^{3} \cdot$ Achim Bub $^{5} \cdot$ Sabine E. Kulling ${ }^{1}$
}

Published online: 11 May 2016

(C) Springer-Verlag Berlin Heidelberg 2016

\section{Erratum to: Arch Toxicol \\ DOI 10.1007/s00204-016-1663-5}

Unfortunately, in lines 12 and 16 of the abstract section, the chemical compound names of the major phase II metabolites of daidzein and genistein were incorrectly published as 7 -sulfo- $4^{\prime}$-glucuronides. The correct names are $4^{\prime}$-sulfo-7-glucuronides.

The online version of the original article can be found under doi:10.1007/s00204-016-1663-5.

Sabine E. Kulling

sabine.kulling@mri.bund.de

1 Department of Safety and Quality of Fruit and Vegetables, Max Rubner-Institut, Haid-und-Neu-Straße 9,

76131 Karlsruhe, Germany

2 Max Planck Institute of Molecular Cell Biology and Genetics, Dresden, Germany

3 Department of Molecular and Cellular Sports Medicine, Institute for Cardiovascular Research and Sports Medicine, German Sport University, Cologne, Germany

4 Molecular Cell Physiology and Endocrinology, Institute for Zoology, Technische Universität Dresden, Dresden, Germany

5 Department of Physiology and Biochemistry of Nutrition, Max Rubner-Institut, Karlsruhe, Germany 